\title{
Optimal Operation of Paralleled Power Transformers
}

\author{
David Trebolle $^{1}$ and Baudilio Valecillos ${ }^{2}$ \\ ${ }^{1}$ Union Fenosa Distribución, Spain \\ ${ }^{2}$ Universidad Carlos III de Madrid, Spain \\ *Email: dtrebolle@unionfenosa.es
}

\begin{abstract}
Parallel operation of power transformers is a common practice. Interest is placed on minimizing the reactive current circulation between transformers due to mismatching of electrical properties. Several control schemes have been applied over the years to ensure efficient operation of paralleled transformers with OLTC. These methods rely either on field measurements of circulating currents or on classic Master/Follower approach and usually assume that the paralleled transformers have matching impedances and OLTC positions. On the present work a generalized approach is implemented calculating the circulating current and minimizing losses directly at the System Operation Office and using the distribution system SCADA to control tap position of the paralleled transformers. This permits the determination of optimal tap matching between transformers and calculation of losses under emergency situations where coupling of transformers with slight impedance mismatch might be considered. In the proposed method a map of circulating current is calculated based on the transformer impedance, $\mathrm{X} / \mathrm{R}$ ratio and OLTC positions, then the optimal tap positions (minimizing losses) under parallel operation are determined.
\end{abstract}

\section{INTRODUCTION}

It is common practice to operate power transformers in parallel and it is usually due to one of the following reasons.

1. Increased Load: If the power of a substation must be increased due to load, one of the economically sound solutions is to add a second transformer in parallel operation.

2. Flexible operation: paralleled transformers can be operated in several ways ensuring reliability, safety, critical load selection and ease of maintenance routine without service outage.

3. High Power: Due to transport size/weight restriction, it may be the only solution to high power requirements.

Even so paralleled operation of power transformers has several drawbacks: increasing short-circuit currents that increase necessary breaker capacity, circulating currents that diminish load capability and increased losses.
The present work focuses on reducing circulating current while analyzing parallel operation of transformers with slightly different impedance, ratio or tap positions.

Previous works in the area are focused on paralleling schemes applied as automatic control of the on load tap changer at the substation, among the more commonly used are the master/follower and circulating currents method [1],[2].

\section{PARALLELED OPERATION OF POWER TRANSFORMER}

There are several conditions that must be met to operate transformers in parallel, some of these conditions are convenient and some are mandatory.

Among the convenient are: same voltage ratio, same short circuit voltage in p.u., and same short circuit power.

The mandatory conditions are: same connection index, and same phase sequence.

When the convenient conditions are not met paralleled operation is possible but not optimal, not complying with mandatory conditions imply phase to phase failures so operation in parallel is not feasible.

\subsection{Ideal working condition.}

In ideal working condition the paralleled transformers will have the same voltage ratio, shortcircuit voltage and short-circuit power. As seen on figure 1 , this implies that:

$I_{a} Z_{c c a}=I_{b} Z_{c c b}=\ldots=I_{N} Z_{c c N}$

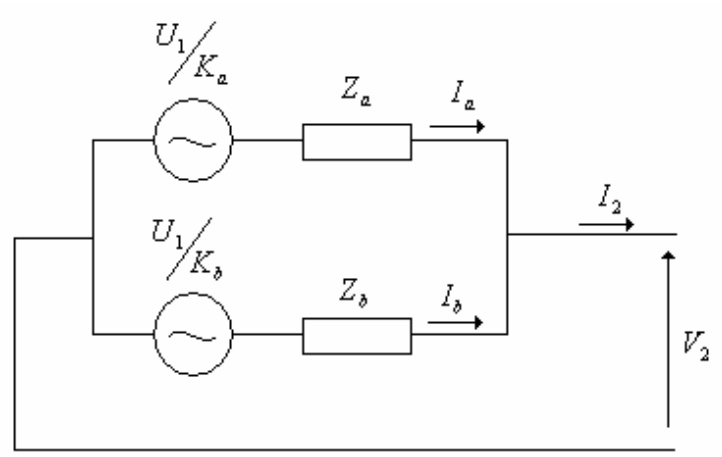

Figure 1. 
Equation (1) can be rearranged dividing by nominal current and voltage as:

$$
\frac{I_{a}}{I_{n a}} \frac{I_{n a} Z_{c c a}}{U_{n}}=\frac{I_{b}}{I_{n b}} \frac{I_{n b} Z_{c c b}}{U_{n}}=. . \frac{I_{N}}{I_{n N}} \frac{I_{n N} Z_{c c N}}{U_{n}}
$$

Defining:

$$
K_{C a}=\frac{I_{a}}{I_{n a}}
$$
that:

As the load ratio of transformer "a" and realizing

$$
U_{c c a \%}=\frac{I_{n a} Z_{c c a}}{U_{n}}
$$

Equating 2 and 4:

$$
K_{C a} U_{c c a \%}=K_{C b} U_{c c b \%}=\ldots=K_{C N} U_{c c N \%}
$$

Equation (5) shows that since all short-circuit voltage is the same in p.u. all transformers will have the same loading ratio, by practical means the paralleled transformer will act as an equivalent having nominal power equal to the sum of the individual transformers and a short-circuit reactance in p.u. equal to the one of is constituents. It should be also clear that power will be shared according to nominal power so each transformer has the same load ratio.

\subsection{Different short-circuit impedance.}

If the transformers to be operated in parallel have different short-circuit impedance in p.u. (1) still holds true but the equivalent impedance shall be determined as:

$$
\frac{1}{Z_{e q}}=\sum_{j=a}^{N} \frac{1}{Z_{c c j}}
$$

The loading ratio of each transformer will be determined by (7)

$$
I_{i}=I_{2} \frac{Z_{e q}}{Z_{c c i}}=I_{2} \frac{1}{Z_{c c i} \sum_{j=a}^{N} \frac{1}{Z_{c c j}}}
$$

Equation 7 can also be rewritten in terms of the equivalent transformer taking into account some definitions:

Nominal power of the group:

$$
S_{n G}=\sum_{j=a}^{N} S_{n j}
$$

Nominal current of the group:

$$
I_{n G}=\frac{S_{n G}}{\sqrt{3} U_{n 2}}
$$

Load ratio of the group:

$$
K_{c G}=\frac{I_{2}}{I_{n G}}
$$

Equating (4) and (7) and after some rearrangement one can arrive at (11) which gives the expression for the short-circuit voltage of the group of transformers operated in parallel, the same result can be obtained using (6) and applying a new per unit base equal to that of the defined quantities of the equivalent group.

$$
U_{c c G \%}=\frac{S_{n G}}{\sum_{j=a}^{N} \frac{S_{n j}}{U_{c c j \%}}}
$$

Also from (7) and (11) the relationship between group loading and transformer loading can be obtained as (12) which is and expression that quickly determines the loading of any transformer within the group from the total load.

$$
K_{c i}=K_{c G} \frac{U_{c c G \%}}{U_{c c i} \%}
$$

From (11) can be seen that if all the transformers have the same short-circuit impedance the equivalent will also have the same short-circuit impedance as stated earlier and the loading ratio of all transformers will be the same. But as short-circuit impedances differ, so will the loading ratio of each transformer of the group. Equation (12) shows that if a transformer has a short-circuit impedance lower to that of the group it will have higher load ratio and that if the transformer has a higher short-circuit impedance that the group it will have a lower load ratio that the group. It can also be shown that if no transformer is to be overloaded the higher loading of the group will be determined by (13) and the maximum power by (14).

$$
\begin{aligned}
& K_{c G \text { max }}=\frac{U_{c c \min }}{U_{c c G}} \\
& S_{G \text { max }}=S_{G n o m} K_{c G \text { max }}=U_{c c \text { min }} \sum_{j=a}^{N} \frac{S_{n j}}{U_{c c j}}
\end{aligned}
$$


The presiding analysis of paralleled operation always takes into account that the transformers have the same voltage ratio.

\subsection{Different voltage ratio.}

Transformers of different voltage ratio have a circulating current which is determined as follows.

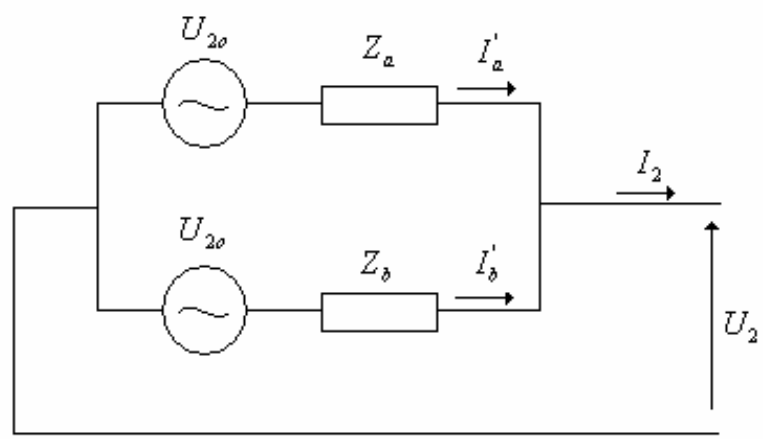

Figure 2.

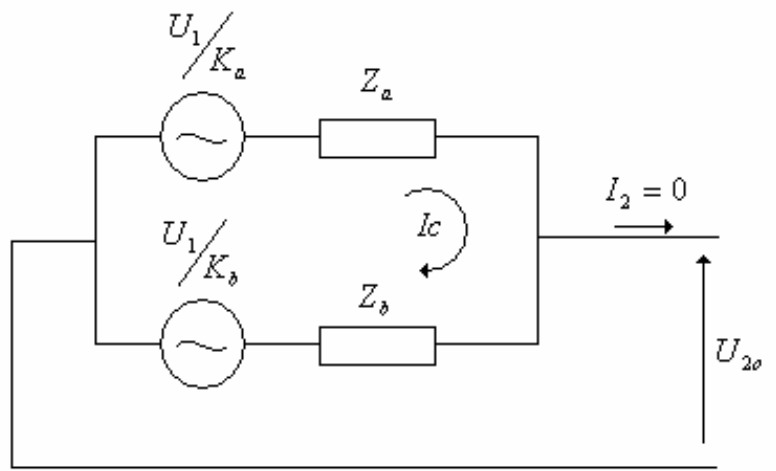

Figure 3.

As the transformers now have a different voltage ratio it can be defined:

$$
\begin{gathered}
U_{1 a}^{\prime}=\frac{U_{1}}{K_{a}} \\
U_{1 b}^{\prime}=\frac{U_{1}}{K_{b}}
\end{gathered}
$$

Where $\mathrm{Ka}$ is the voltage ratio of transformer " $\mathrm{a}$ " $\mathrm{Kb}$ the voltage ratio of transformer " $b$ " and for the sake of argument it will be assumed that $K_{a}<K_{b}$ which implies that $U_{1 a}^{\prime}>U_{1 b}^{\prime}$. Then the circulating current can be calculated as (17).

$$
I C=\frac{\frac{U_{1}}{K_{a}}-\frac{U_{1}}{K_{b}}}{Z_{a}+Z_{b}}=\frac{U_{1}}{K_{a}} \frac{\left(1-\frac{K_{a}}{K_{b}}\right)}{Z_{a}+Z_{b}}
$$

In practice the short-circuit impedance are around $10 \%$, so small differences between voltage ratios will produce large circulating currents, transformers of the same power, short circuit impedance of $10 \%$ and a voltage ratio difference of $10 \%$ will have almost full nominal current circulating without any load.

\subsection{Different short-circuit impedance and voltage ratio.}

To study the behaviour of paralleled transformers including load and circulating current, superposition can be applied. Figures 2 and 3 represent each of the cases.

First, figure 2 where there is a load current without circulating current and then in the figure 3 the circulating current without the load. Defining the load current trough transformer "a" and "b" as $I_{a}^{\prime}$ and $I_{b}^{\prime}$, they can be obtained using (7), the result in (18) and (19).

$$
\begin{aligned}
& I_{a}^{\prime}=I_{2} \frac{Z_{b}}{Z_{a}+Z_{b}} \\
& I_{b}^{\prime}=I_{2} \frac{Z_{a}}{Z_{a}+Z_{b}}
\end{aligned}
$$

The full current can be calculated adding the load current to the circulating current as shows on (20) and (21).

$$
\begin{aligned}
& I_{a}=I_{a}^{\prime}+I C \\
& I_{b}=I_{b}^{\prime}-I C
\end{aligned}
$$

In real systems is usual to keep the difference in short-circuit reactance under $7.5 \%$ in nominal tap position, even so differences in tap operation may increase this reference value and also change the nominal voltage of the transformers[3]. The classic formulation for determining circulating reactive current in paralleled transformers has been explain in detail in the previous section [4],[5]. Using the presiding formulae operational limits are defined for the operation on paralleled transformers with on load tap changers. Differences arising in the short-circuit reactance due to tap change are neglected and taps are treated only as originating a different voltage ratio within coupled transformers. 


\subsection{Application}

Using the previous formulation an application has been developed, which allows for calculating a current map that shows the circulating current, total current, load ratio, etc. of any pair of transformers selected from an available database. In figure 4 the main windows application program providing all the data relevant to transformer coupling. Two transformers of the following characteristics are shown; they are feeding a total load of 30MVA, 10MVAr.

Transformer A:

$$
\begin{aligned}
& V_{\text {na }}=130 \mathrm{kV} \pm 10 \% / 45 \mathrm{kV} \\
& S_{\text {na }}=30 M V A \\
& Z_{\text {сса } \%}=12.58 \% \\
& n^{\circ} \mathrm{TAP}=21
\end{aligned}
$$

Transformer B:

$$
\begin{aligned}
& V_{\text {na }}=132 \mathrm{kV} \pm 12 \% / 46 \mathrm{kV} \\
& S_{\text {na }}=60 \mathrm{MVA} \\
& Z_{\text {сса } \%}=11.5 \% \\
& n^{\circ} \mathrm{TAP}=21
\end{aligned}
$$

In figure 5 is the current map showing that the minimum circulating current is not always achieved operating the transformers in the same tap position. These optimal operating positions can be then downloaded to the distribution system SCADA and automatically match tap position to minimum circulating current. The application is also used to determine maximum tap range excursion allowed to OLTC transformers coupled with no-load tap transformers.

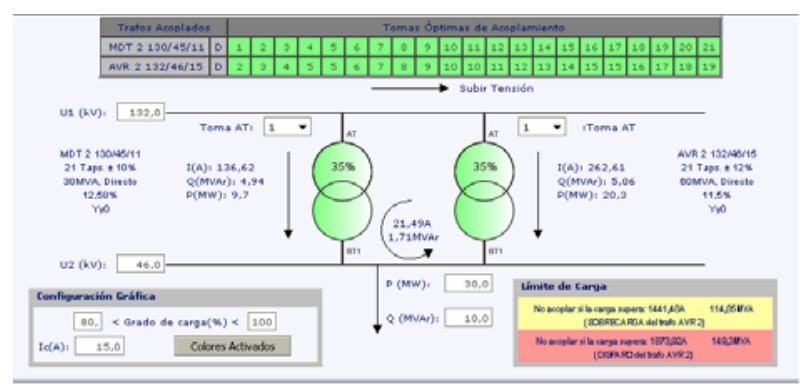

Figure 4.

The application developed is also used as training tool for the system control office, used to highlight the importance of proper tap selection during paralleled operation. For instance in table I several typical values are presented as a function of transformer ratio. In the second column the MVAr circulating between the transformers as per one tap position difference. In the third column the number of taps mismatch that will generate a circulating VAr flow equal to the nominal power. The fourth column shows the percentage of circulating MVAr to nominal power at extreme tap position. This shows the importance of proper tap selection, and how the circulating current due to tap position mismatch can be enormous.

\begin{tabular}{|c|c|c|c|}
\hline $\begin{array}{l}\text { Nominal Voltage } \\
\text { Ratio }\end{array}$ & Qc/tap (MVAr/tap) & Taps (Qc=Snom) & $\begin{array}{l}\text { Qc extrem } \\
\text { tap/Snom (\%) }\end{array}$ \\
\hline $220 / 45$ & 7 & 13 & 161 \\
$220 / 15$ & 1.2 & $\infty$ & 25 \\
$132 / 45$ & 3.5 & 21 & 108 \\
$132 / 15$ & 1.5 & $\infty$ & 97 \\
$66 / 15$ & 1.2 & 21 & 100 \\
$45 / 15$ & 2 & 15 & 156 \\
\hline
\end{tabular}

Table I.

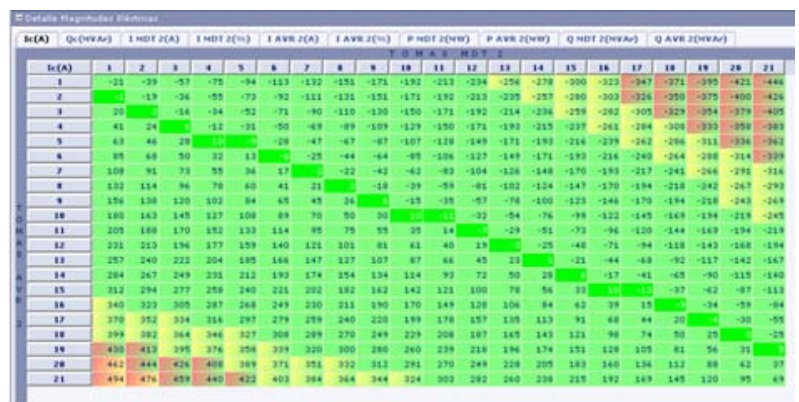

Figure 5.

Also a batch process is implemented applying the previous formulas to all the transformers within Unión FENOSA prefiltered to a maximum of $10 \%$ nominal voltage difference and the circulating current are calculated for every tap position. The results of this are maps of circulating current for each possible pair of transformers feasible of being operated in parallel. Then a risk map is associated to the transformers and possible paralleling partner are determined by colour as show in figure 6. Not likely to operate in parallel is shown as red. Likely operation but suboptimal is indicated as yellow. Operation with circulating current under a predefined value for every (adequate) tap combination is green. This is done in order to provide with guidelines to planning and maintenance subsections about future movement of transformers within the network.

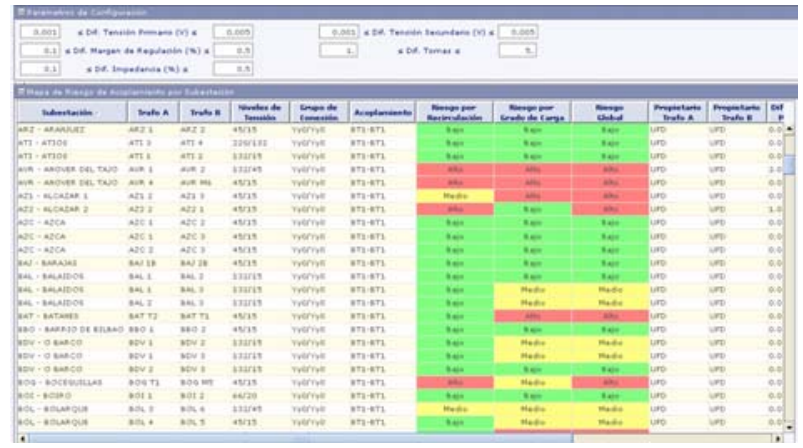

Figure 6. 


\section{CONCLUSIONS}

A detail explanation of the calculations need to couple power transformers for parallel operation has been deduced, these formulae are later employed to develop an application that analyzes transformer paralleling and determines optimal tap setting by simple multiple function evaluations. This information is then used to remotely control tap changer position of in field transformers trough SCADA to minimize circulating currents and reduce losses. Also a batch process is applied that yields useful information about transformers feasible of paralleled operation. This information is later used to bias transformer relocation to substations where they have possible paralleling partners.

\section{REFERENCES}

[1] E. Tom Jauch, "Factors in Choosing Transformer Paralleling Methods", IEEE, PES T\&D 2005/2006 Dallas. TX.

[2] James H. Harlow, "Let's Rethink Negative Reactance Transformer Paralleling", Transmission and Distribution Conference and Exposition, 2003 IEEE PES, 7-12 Sept. 2003, Volume: 2, On page(s): 434438 vol.2

[3] P. Okanik, B. Kurth, J.H. Harlow, "An Update on the Paralleling of OLTC Power Transformers". 07803-5515-6/99/\$10.00 0 1999 IEEE

[4] Martin J. Heathcote, “J\&P Transformerbook" Thirteenth edition, 2007, Newnes, pag. 470-481.

[5] Jesús F.Mora, "Maquinas eléctricas” Fiveth edition. 2003 Mc Graw Hill. Pg, 227-230. 